Subjects and methods 135 patients (Beijing Tong Ren Hospital)were enrolled (male, 96, female, 39; ages 65 \pm 13 ) They were divided into three groups: DM patients $(n=77)$, IGT patients $(n=38)$ and controls $(n=20)$. The level of serum YKL-40 was measured by ELLISA. Lipids, $\mathrm{HbA}_{1} \mathrm{C}$, plasma glucose and serum high-sensitivity C-reactive protein (hs-CRP) were detected by routine methods. Date was performed on High-resolution B-model ultrasound. Data was analysed using SPSS16.

Results (1) Serum YKL-40 levels in DM group was higher than that in control group $(p=0.02)$. It was higher in IGT group than that in control group, but without statistically significance $(p=0.08)$. It was of no significant difference between DM and IGT group $(p=0.06)$. (2) IMT was different among three groups $(p<0.05)$. (3) The serum YKL-40 level was associated with DM $(B=-0.08, p=0.007$, OR 0.992). After adjusting age, gender, creatinine and the risk factors of $\mathrm{CAD}$, diabetic family history, hypertension, smoking and hs-CRP, it was still associated with $\mathrm{DM}(\mathrm{B}=-0.009, \mathrm{p}=0.01$, OR 0.991). (4) The serum YKL-40 level was associated with DM combined with atherosclerosis $(B=-277, p=0.026$, OR 1.319). (5) There was a notable correlation between the serum YKL-40 levels and IGT with cardiovascular risks $\left(\mathrm{X}^{2}=6.96, \mathrm{p}=0.016\right)$.

Conclusions (1) The level of serum YKL-40 was increased in patients with DM and IGT. (2) The level of serum YKL-40 was associated with diabetes. (3) The level of serum YKL-40 may be used as an independent predicted factor for cardiovascular risk in IGT.

\section{[gw22-e0199] STUDY OF SERUM YKL-40 LEVEL AND IMPAIRED GLUCOSE TOLERANCE COMBINED WITH CARDIOVASCULAR DISEASES RISK}

Ren Jie, Qin Minzhao Beijing Tongren Hospital, Beijing, China

10.1136/heartjnl-2011-300867.347

Background and Objective Diabetes has become a common healthy problem in the world, which is an autoimmune disease mediated by inflammatory cytokine. Inflammation plays an important role in the pathogenesis of diabetes and macrovascular complications. Chitinase-3-like protein-1 (YKL-40) is a new inflammatory factor, which can influence the level of other inflammatory markers and related to the development of cardiovascular diseases. This study aims to investigate the level of YKL-40 in patients with diabetes and impaired glucose tolerance. Correlation between YKL-40 and blood glucose, lipids, high sensitivity C-reactive protein, IMT was compared. 